\title{
Accidental Olanzapine Consumption in Children
}

\author{
Kumar JK ${ }^{1}$, Samatha $A^{2}$, Marda $S^{3}$, Chaudhary $D^{4}$
}

\begin{abstract}
Olanzapine is commonly used an atypical antipsychotic drug. Overdose is characterized by agitation, deep coma, blurred vision, myosis, respiratory depression and cardiovascular effects. Cardiovascular toxicity includes alterations in blood pressure, conduction disturbances like prolongation of the QRS/ QT intervals or ventricular dysrhythmias. We report two children with Olanzapine poisoning who presented with altered consciousness and one of them had ECG abnormality. Both of them recovered and discharged.
\end{abstract}

Key words: Olanzapine overdose, altered consciousness, ECG abnormality.

\section{Introduction}

$\mathrm{O}$ lanzapine is commonly used drug in children for psychiatric illness ${ }^{1}$. It is an atypical antipsychotic drug and its clinical utility has increased in the last twenty years ${ }^{2}$. As the prescription of this medication in adults increases, the frequency of unintentional paediatric poisonings will also increase ${ }^{2}$. Overdose means ingestion of a drug in quantities greater than recommended. Overdose may result in toxic state and death. It is a pharmacological concept. Poisoning means organism being exposed to chemicals, plants, and other toxic substances that can cause harm. It occurs when the material interferes with normal body functions. Poisoning is clinical. In children Olanzapine overdose is more associated with significant adverse effects and is characterised by agitation, deep coma, blurred vision, myosis, respiratory depression, cardiovascular effects, hypotension, extrapyramidal and anticholinergic effects ${ }^{2,3}$. We are reporting two children with Olanzapine poisoning who presented to us on the same day.

\section{The Cases}

Case 1: A two years age boy brought with altered sensorium since three hours after accidental consumption of two tablets (10 $\mathrm{mg}$ each) of Olanzapine. On examination, child was unconscious [GCS 6/15] with constricted pupils. His temperature $98.4^{\circ} \mathrm{F}$, Pulse Rate 116/minute, Respiratory Rate 22/minute, BP 90/60 mm of $\mathrm{Hg}$, CFT $<3$ seconds and oxygen saturation $99 \%$ with room air. His blood counts, LFT, RFT, ABG, blood sugar, serum calcium and magnesium were normal. His ECG showed heart rate of 120/minute, normal PR \& QT interval, conduction disturbance in terms of RsR pattern [Fig
${ }^{1}$ Dr. K. Jagadish Kumar MBBS, MD, Professor of Paediatrics. ${ }^{2} \mathrm{Dr}$ Aleti Samatha, MBBS, MD Resident in Paediatrics. ${ }^{3} \mathrm{Dr}$. Srikanth Marda, MBBS, MD Resident in Paediatrics. ${ }^{4}$ Dr. Divya Chaudhary, MBBS, MD Resident in Paediatrics. All From The Jagadguru Sri Shivarathreeshwara Medical College (JSSMC), Jagadguru Sri Shivarathreeshwara (JSS) University, Mysore, India

\section{Address for correspondence \\ Dr. K. Jagadish Kumar \\ E-mail: jagdishmandya@gmail.com}

\section{How to cite}

Kumar JK, Samatha A, Marda S, Chaudhary D. Accidental Olanzapine Consumption in Children. J Nepal Paediatr Soc 2017;37(3):267269.

doi: http://dx.doi.org/10.3126/jnps.v37i3.16384

This work is licensed under a Creative Commons Attribution 3.0 License.

\section{(c) (i)}


1]. Stomach wash and activated charcoal given along with maintenance intra venous fluids. He regained consciousness by eight hours of admission. His ECG after 36 hours was normal and was discharged.

Case 2: Developmentally normal eight year age boy brought with excessive drowsiness since five hours. There was no history of fever, vomiting, convulsions, unknown bite or trauma. On examination his vitals were stable. His pupils were constricted, GCS 9/15, nervous system examination did not reveal any focal deficits. Other systems examination were normal. His blood counts, LFT, RFT, blood sugar, serum cholinesterase, serum calcium and magnesium were normal. His CT scan brain and ECG was normal. Lumbar puncture was traumatic. He became conscious by six hours and revealed ingestion of two tablets of Olanzapine $(15 \mathrm{mg}$ each) which father was taking for psychiatric illness. He was treated symptomatically and discharged after 36 hours.
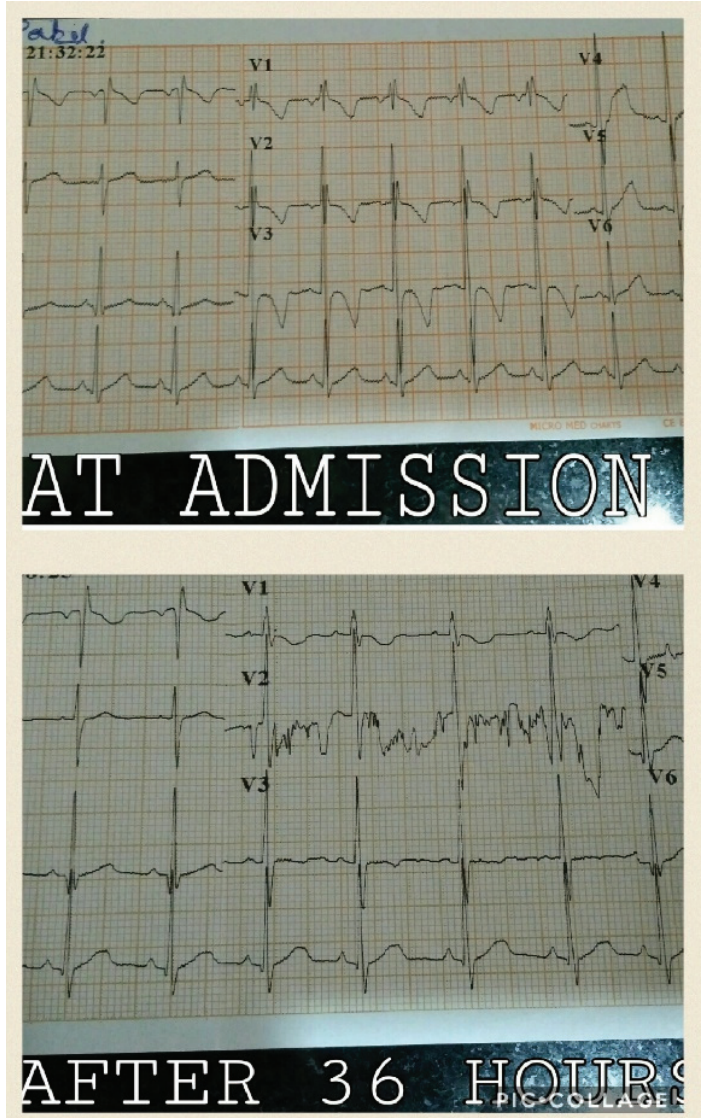

Fig 1: ECGs at admission and before discharge

\section{Discussion}

Olanzapine is an atypical antipsychotic drug commonly used in paediatrics considering its lower incidence of extrapyramidal adverse effects than typical antipsychotics ${ }^{1,2}$. Overdose is characterized by agitation, deep coma, blurred vision, myosis, respiratory depression, cardiovascular effects, hypotension, extrapyramidal and anticholinergic effects ${ }^{2,3}$. Systemic review of safety of Olanzapine in young children by Flank et al reported sedation in $78 \%$, extrapyramidal symptoms in $9 \%$, electrocardiogram abnormalities $14 \%$ and elevation in liver function tests in $7 \%$.There was no deaths ${ }^{1}$. Cardiovascular toxicity is more with typical antipsychotics than with atypical antipsychotic drugs $^{4,5}$. It includes alterations in blood pressure, conduction disturbances like prolongation of the QRS/ QT intervals or ventricular dysrhythmias ${ }^{4}$. Systematic review of cardiovascular effects following atypical antipsychotic medication overdose by Tim et al revealed nine paediatric patients with olanzapine overdose. Out of them six had tachycardia, two developed hypotension and one of them treated with vasopressors. However there was no ventricular arrhythmias, prolongation of QT/ QRS interval or deaths. But adult patients had all these abnormalities and deaths also due to olanzapine overdose $^{4}$. Ciszowski et al observed prolonged QTC and supraventricular tachycardia (including sinus tachycardia) in $22 \%$ of 23 acute olanzapine poisonings in adults. They also noted ST-T changes, supraventricular premature \& ventricular premature complexes, bundle branch block, sinus bradycardia and atrial fibrillation ${ }^{6}$. Our patient also had tachycardia and conduction disturbance like $R s R$ pattern.

As the usage of Olanzapine increases in adults, the frequency of unintentional paediatric ingestions will also increase like in our cases ${ }^{2}$. The toxic dose for olanzapine is $0.5 \mathrm{mg} / \mathrm{kg}$ and can cause severe effects that last 1-3 days, often with one tablet in children ${ }^{2}$.Our first child had ingested $1.6 \mathrm{mg} / \mathrm{kg}$ and another $1.2 \mathrm{mg} /$ $\mathrm{kg}$ of Olanzapine. In terms of over dosage, Olanzapine appears to be a medium-level safe medicine ${ }^{2}$. Olanzapine overdose mimic opiate intoxication ${ }^{2}$. Therefore olanzapine intoxication should be considered in the differential diagnosis of the patient with depressed mental status and meiosis along with opioid ${ }^{2}$. Both of our children presented with altered consciousness and meiosis on the same day.

Venkata et al reported 15 year boy who presented with agitation, constricted pupils and a GCS fluctuating between 6 to 11. His ECG showed sinus tachycardia, tall T wave with ST depression. Retrospectively history of olanzapine overdose was revealed similar to our patient ${ }^{5}$.

\section{Conclusions}

In view of increasing use of Olanzapine in recent times, overdose with Olanzapine should be considered in the differential diagnosis of children presenting with unexplained drowsiness, meiosis and ECG changes. 


\section{References}

1. Flank J, Sung L, Dvorak CC, Spettigue w, Dupuis LL. The Safety of Olanzapine in Young Children: A Systematic Review and Meta-Analysis. Drug Saf 2014;37:791-804.

2. Dokur M, Gülmez GP, Kuzudişli SU. Clinical Findings of Emergence After Olanzapine Overdosage: Pediatric Case Report and Review of Literature. JAEMCR 2014;5:100-6.

3. Singh LK, Praharaj SK, Sahu M. Nonfatal Suicidal Overdose of Olanzapine in an Adolescent. Current Drug Safety 2012;7:328-329.
4. HH, Hoppe J, Heard K. A systematic review of cardiovascular effects following atypical antipsychotic medication overdose. Am J Emerg Med 2009;27:60716.

5. Venkata RN, Vaswani J. Case reports of atypical (second generation) antipsychotic overdose in adolescents. Arch Dis Child 2012;97:A73 doi:10.1136/ archdischild-2012-301885.177.

6. Ciszowski K, Sein Anand J. Electrocardiographic abnormalities in acute olanzapine poisonings. Przegl Lek 2011;68:422-25. 